\title{
Early relapse is an adverse prognostic marker in systemic immunoglobulin light chain (AL) Amyloidosis
}

\author{
Sriram Ravichandran (D) $^{1}$, Steven Law ${ }^{1}{ }^{1}$, Shameem Mahmood ${ }^{1}$, Brenden Wisniowski ${ }^{1}$, Darren Foard ${ }^{1}$, Marianna Fontana ${ }^{1}$, \\ Ana Martinez-Naharro ${ }^{1}$, Carol Whelan ${ }^{1}$, Julian D. Gillmore ${ }^{1}$, Helen J. Lachmann ${ }^{1}$, Philip N. Hawkins ${ }^{1}$ and Ashutosh D. Wechalekar (iD ${ }^{\circledR}$
}

(c) The Author(s) 2022

Leukemia (2022) 36:1180-1184; https://doi.org/10.1038/s41375-021-01497-7

\section{TO THE EDITOR:}

Systemic Immunoglobulin light chain amyloidosis (AL) is a proteinmisfolding disorder associated with an underlying monoclonal B-cell or plasma cell dyscrasia. Whilst the prognosis of $\mathrm{AL}$ has markedly improved with novel agents [1], it remains incurable with a relapsing-remitting course, necessitating multiple treatment lines. Baseline cardiac bio-markers [2] and the depth of response to initial therapy [2-4] are critical prognostic variables. Early relapse confers a poor prognosis in Myeloma [5-7]. There is little information on the impact of response durability on outcomes in $\mathrm{AL}$. The dual pathology in $\mathrm{AL}$ (the clonal biology and the organ dysfunction due to amyloid deposition) lead to a complex interplay of factors. We have recently demonstrated that outcomes after each relapse episode are similar, i.e., deaths occur at each relapse [8]. Here, we assess the impact of timing of relapse on outcomes in a cohort of $\mathrm{AL}$ patients treated with upfront Bortezomib.

\section{PATIENTS \& METHODS}

All patients treated with frontline Bortezomib in 2010-2019 at the National Amyloidosis Centre, UK, are included in the analysis. Patients with primary refractory disease, those with $\leq 24$ months follow up and continuing response and those who received 2 nd line therapy for reasons other than progression are excluded from the analysis. We report response (complete response- CR; very good partial response- VGPR; partial response-PR) based on the validated ISA criteria. [2] We report haematologic progression based on the consensus criteria published by the ISA (ISA Criteria, Table SA1) [9]. We also report progression based on the recently published "high-risk" dFLC (difference between involved and uninvolved light chains) criteria (Pavia Criteria, Table SA1) [10]. We define progression as earlier of the two criteria (conventional or high-risk dFLC). We analysed the survival of patients based on the cut-offs reported in Myeloma ( $\leq 12$ months $\& \leq 24$ months). Due to the high and ongoing early mortality from end-organ damage in cardiac AL (even in patients in a deep response as we and other authors have previously reported), which persists at 12 months from diagnosis $[8,11]$, to keep the focus on the impact of clonal progression in this manuscript and to avoid introducing a bias due to the organ related mortality independent of progression, we chose 24 months (Early relapse, ER) as the optimal cut-off for this analysis. Late relapse (LR) was defined as patients who either had haematologic progression after 24 months or had not relapsed beyond 24 months to the last follow up. A detailed description of the patients and methods is available in the Supplementary Appendix.

\section{RESULTS \& DISCUSSION}

In total, 560 patients are analysed here (Fig. SA1). In total, 513/560 $(91.6 \%)$ patients were treated with CyBorD. The other regimens were-Bortezomib-Dexamethasone (4.5\%), Bortezomib-ThalidomideDexamethasone (2.1\%), Bortezomib-Melphalan-Prednisolone $(0.7 \%)$, Bortezomib-Adriamycin-Dexamethasone (0.5\%), BortezomibRituximab-Dexamethasone (0.4\%) and Bortezomib-LenalidomideDexamethasone $(0.2 \%)$.

$331 / 560(59.1 \%)$ patients fulfilled one of the two progression criteria (ISA or Pavia criteria). 267 patients fulfilled the ISA progression criteria, and 235 patients fulfilled the Pavia criteria (Figs. SA2 \& 3). 171/331 (51.7\%) patients had progressed by both criteria at the time of this analysis. 64/331 (19.3\%) had only progressed by the Pavia criteria, and 96/331 (29\%) had only progressed by the ISA criteria. Of the 171 patients who progressed by both criteria, $73 / 171$ (42.7\%) fulfilled both criteria at the same time, 44/171 (25.7\%) patients progressed by the ISA criteria before the Pavia criteria (median difference between the two progression criteria was 4 months, range 1-28 months) and 54/171 (31.6\%) progressed by the Pavia criteria before the ISA criteria (median difference 3.5 months, range 1-22 months).

$250(44.6 \%)$ and 310 (55.4\%) patients had early (ER) and late (LR) relapse, respectively. In the LR group, $81(26.1 \%)$ patients had progressed, and $229(73.9 \%)$ patients were in continuing response. $38 / 331(11.5 \%)$ patients had died after progression without receiving further therapy (Table SA2 and Fig. SA4). 40/560 (7.1\%) patients received a stem cell transplant. The baseline characteristics of the two groups are captured in Table 1 (significant differences in bold). The ER group had more advanced cardiac disease $(p<0.005)$, a higher serum M-protein $(p=0.023)$ and higher dFLC $(p<0.005)$. The median bone marrow plasma cell

${ }^{1}$ National Amyloidosis Centre, University College London (Royal Free Campus), London, UK. ${ }^{凶}$ email: a.wechalekar@ucl.ac.uk 


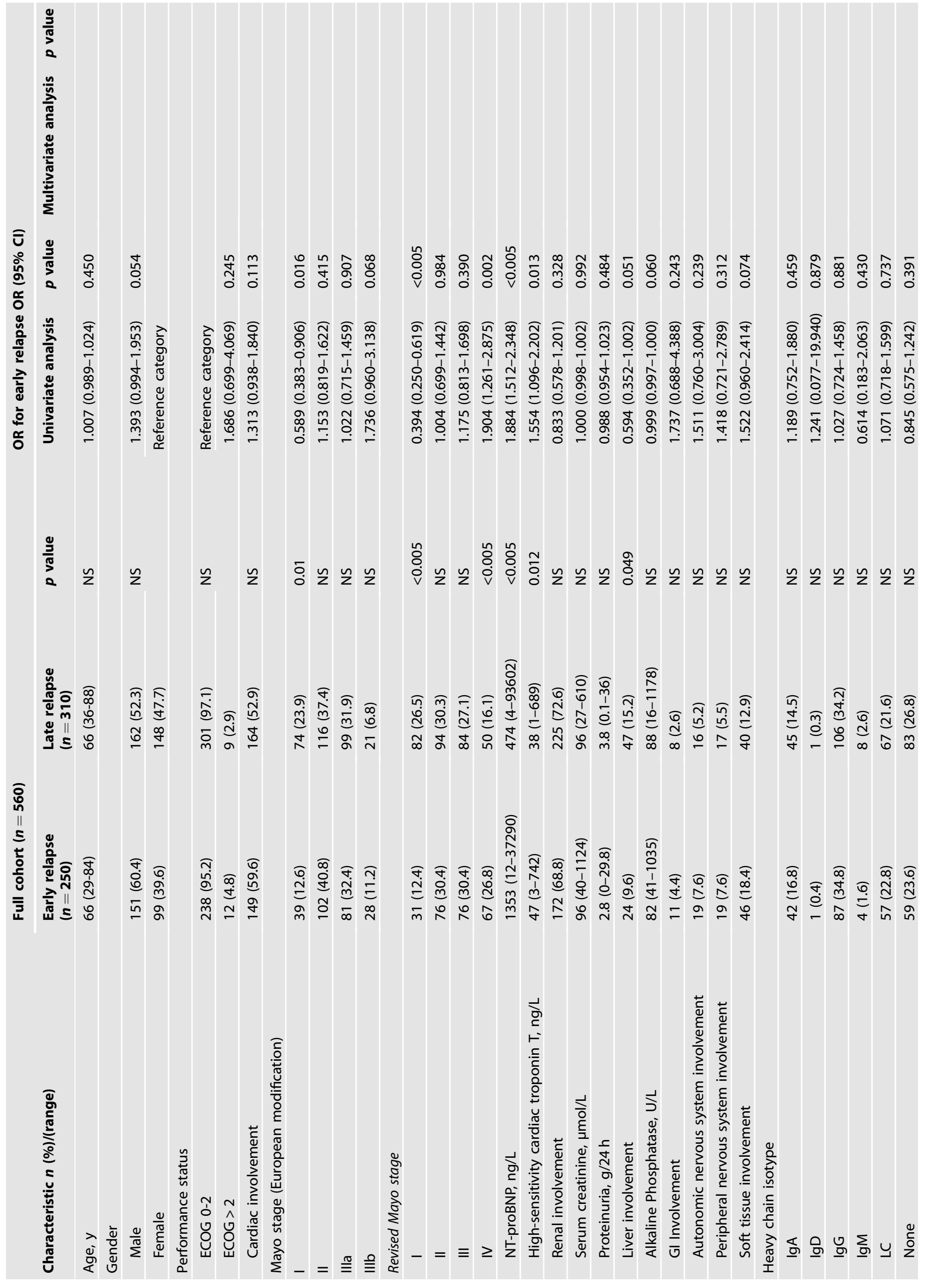




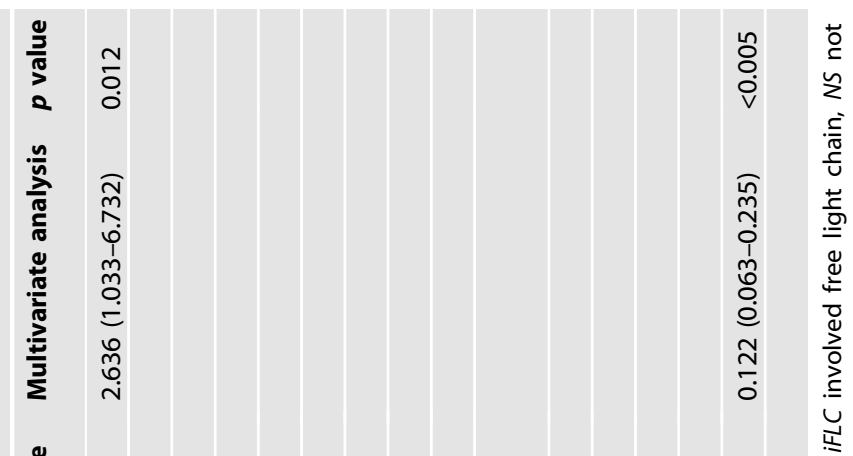

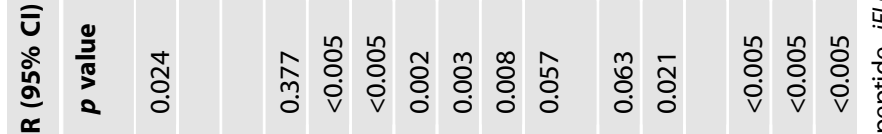
5

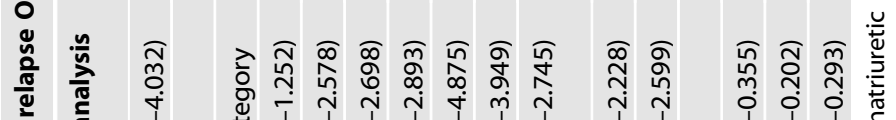

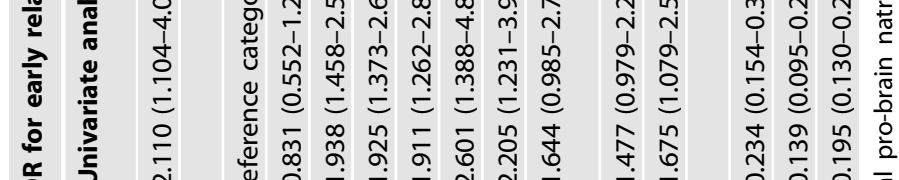

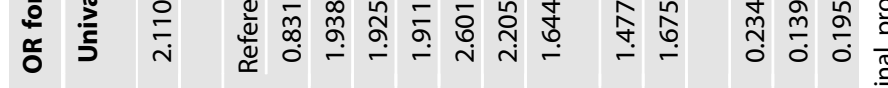

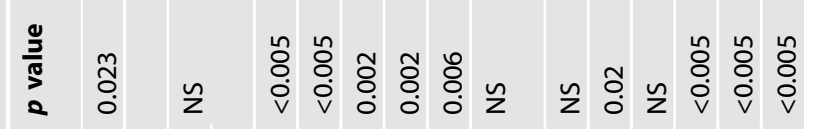

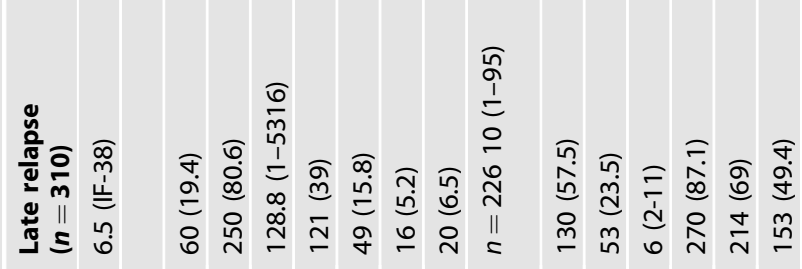

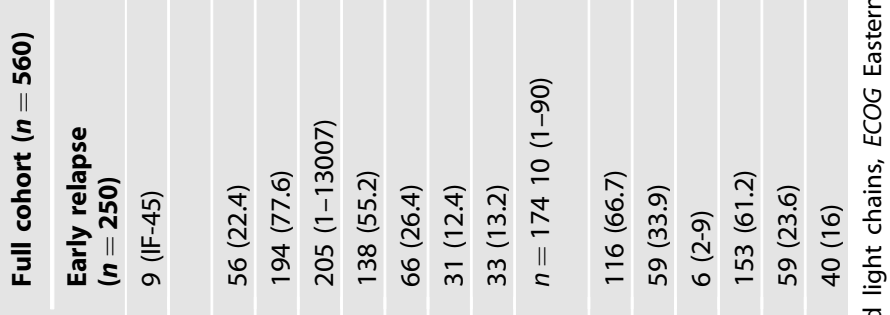

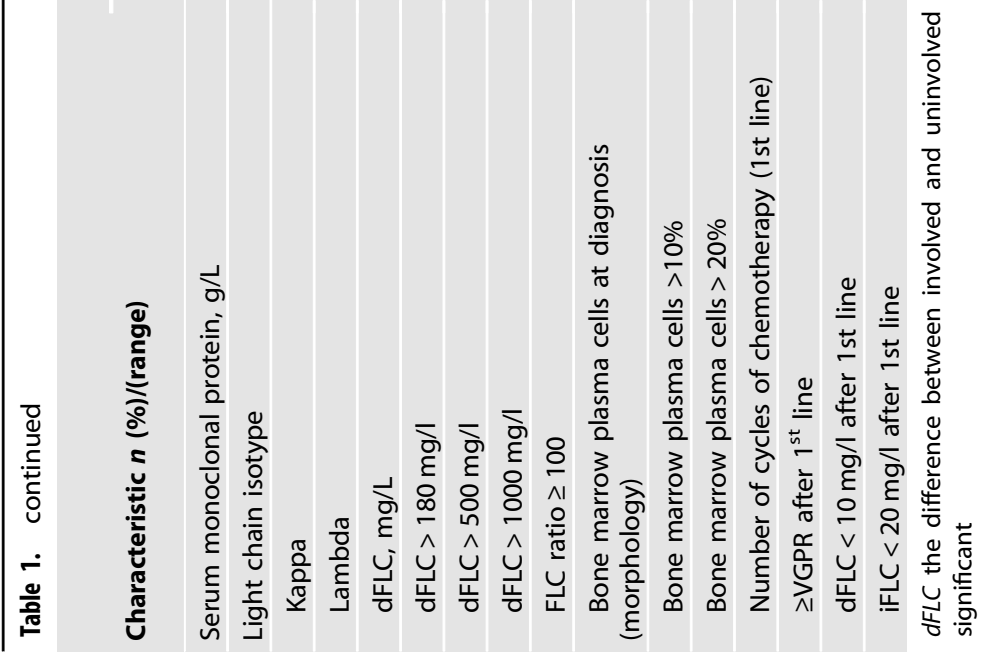


A

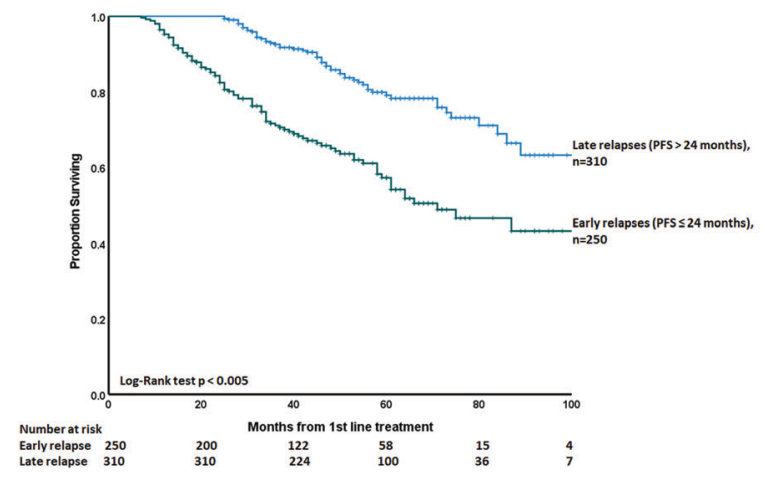

B

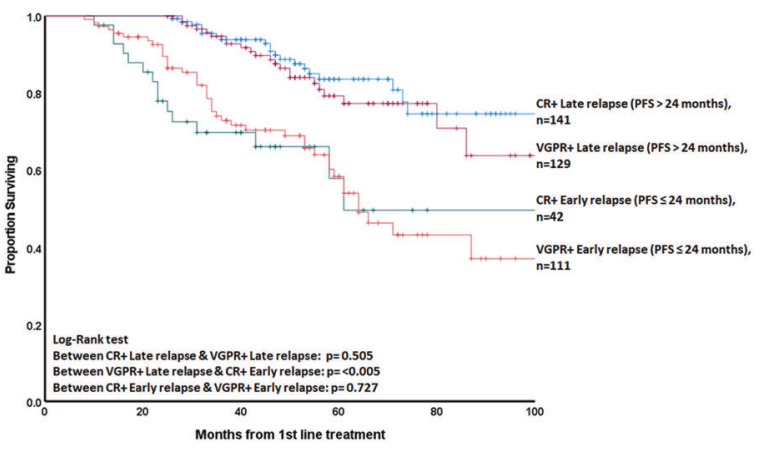

Fig. 1 Impact of early relapse on overall survival. A Kaplan-Meier curve showing the impact of early ( $\leq 24$ months) vs late ( $>24$ months) relapse on OS from 1 st line. Patients with early relapse had a significantly poorer survival than the late relapses-median OS 71 months $(95 \%$ Cl 53.53-88.46 months) vs not reached ( $p<0.005)$. $100 \%, 100 \%$ and $80 \%$ of LR patients were alive at the end of $1,2 \& 5$ years, respectively. B Kaplan-Meier curve showing the impact of early ( $\leq 24$ months) vs late ( $>24$ months) relapse on OS from $1 s t$ line, stratified by the haematologic response (CR \& VGPR) after 1st line. Patients who relapsed early had a significantly poorer survival than those who relapsed late, irrespective of their initial haematologic response $(p<0.005)$. There was no significant difference in survival between CR+ early response $\&$ VGPR + early response- median OS 61 months vs 64 months (95\% Cl 53. 91-74.08 months), $p=0.727$. Similarly, there was no significant difference in survival between CR + late response and VGPR + late response- median OS 109 months vs not reached, $p=0.505$. VGPR + late response had a superior survival than CR + early response- median OS not reached vs 61 months, $p<0.005 .0$, $100 \%, 100 \%$ and $80 \%$ of VGPR + late response were alive at the end of 1, $2 \& 5$ years, respectively.

percentage was not significantly different in either group; but, the ER group had a higher proportion of patients with plasma cells $>$ $20 \%$ (33.9\% vs. $23.5 \%, p=0.02$ ). The ER group also had a lower proportion of patients with deep haematologic response after 1st line therapy $(p<0.005)$.

The median OS of the entire cohort was 109 months. Patients with ER had significantly poorer survival than the LR patientsmedian OS 71 months vs not reached after 1 st line $(p<0.005)$ (Fig. 1A). ER patients had poorer survival irrespective of the depth of response to 1 st line therapy $(p<0.005)$. ER patients had poorer survival than LR patients after 2nd line also (Fig. SA5). The outcomes of patients achieving a CR or VGPR were not significantly different within ER or LR groups, but patients with CR/VGPR in the LR group had significantly better survival than patients with $C R$ in the ER group-median OS not reached vs 61 months $(p<0.005)$ (Fig. 1B and SA6).

In a univariate model (Table 1), disease stage [Mayo stage I ( $p=$ $0.016)$, revised Mayo stage I $(p<0.005), \&$ revised Mayo stage IV $(p=0.002)]$, NT-proBNP $(p<0.005)$, Troponin T $(p=0.013)$, serum M-protein $(p=0.024)$, FLC ratio $\geq 100(p=0.008), \operatorname{dFLC}(p<0.005)$, bone marrow plasma cells $>20 \%(p=0.021), \geq$ VGPR after 1 st line $(p<0.005), \mathrm{dFLC}<10 \mathrm{mg} / \mathrm{l}(p<0.005)$, and iFLC $<20 \mathrm{mg} / \mathrm{l}(p<$ 0.005 ) were significant predictors of early relapse. In a Multivariate model including NT-proBNP, Troponin T, baseline dFLC, serum M-protein and bone marrow plasma cells $>20 \%$, serum M-protein [OR $2.460(95 \% \mathrm{Cl} 1.076-5.624), p=0.033]$ \& NT-proBNP [OR 1.506 (95\% Cl 1.036-2.188), $(p=0.032)]$ were significant predictors of early relapse. When haematologic response (dFLC $<10 \mathrm{mg} / \mathrm{l}$ or iFLC $<20 \mathrm{mg} / \mathrm{L}$ (separate models for each respectively)) was added to the above model (instead of the baseline dFLC), we found serum M-protein [OR $2.636(95 \% \mathrm{Cl} 1.033-6.732), p=0.043]$ \& dFLC $<10 \mathrm{mg} / \mathrm{l}$ [OR $0.122(95 \% \mathrm{Cl} 0.063-0.235),(p<0.005)$ ] (as well as iFLC $<20 \mathrm{mg} / \mathrm{L}$ (OR 0.242, 95\% Cl 0.126-0.465, $p<0.005)$ ) were significant predictors of early relapse.

These data show that AL patients with haematologic relapse within 24 months of initial treatment have significantly poorer survival -this appears to be linked to the biology of the underlying clone as the early relapsing patients have higher presenting dFLC, serum M-protein and bone marrow plasma cells. They also have worse organ involvement with higher NT-proBNP at presentation. The clonal biology and the degree of organ involvement are likely related since patients with bone marrow plasma cells $>20 \%$ have a greater cardiac involvement [12]. The depth of response to the initial treatment is a critical determinant of response durability- the ER group had a poorer depth of response to initial chemotherapy, and a deep response ( $\mathrm{dFLC}<10 \mathrm{mg} / \mathrm{L}$ ) was independently predictive of LR. Patients with advanced cardiac involvement are often sicker; inevitably have dose modifications/treatment delays in addition to having a higher clonal burden- both likely to impact haematologic response. The patients in this cohort were treated at their local hospitals, and we do not have access to the dose intensity; therefore, we cannot test the above hypothesis. We acknowledge this limitation of the present data. The present data are congruent with the published data in Myeloma [5-7, 13].

Whilst there are no published reports on response durability impacting outcomes in AL to compare with the current analysis, the Mayo clinic group has reported that patients with higher presenting plasma cell percentages have poorer outcomes [12] and higher cardiac involvement, consistent with the current study. The presence of cytogenetic abnormalities (hyperdiploidy or $t(11 ; 14))$ is associated with poorer outcomes in AL [14]. The lack of cytogenetic data (at presentation and relapse) in the current cohort is a significant limitation.

Since the current cohort was exclusively treated with bortezomib upfront, we cannot comment on the impact of other therapies on ER/LR. As clonal burden at presentation appears to be a marker of early progression, treatment strategies may need modulating on initial clonal markers (in addition to organ involvement that has dominated therapeutic adjustments). As deeper responses translated to LR, Daratumumab-CyBorD, which is highly effective in achieving deep responses, is an attractive option; but, longer follow up is needed for progression data in the Andromeda study [15]. Other options may include deferred autologous stem cell transplantation (SCT) consolidation in those not eligible for an upfront SCT. There is little data on maintenance therapy to delay relapse, and prospective trials are required.

The study's retrospective nature, absence of cytogenetic data at diagnosis and clonal evolution at progression remain limitations of this analysis.

In conclusion, response durability is an equally important variable (along with the depth of response) in assessing prognosis in AL. Regimens capable of inducing a deep response are available 
and should be adopted early. The early relapses should be considered for clinical trials that can identify treatments with the potential to overcome the high-risk biology of the disease.

\section{REFERENCES}

1. Ravichandran S, Lachmann HJ, Wechalekar AD. Epidemiologic and survival trends in amyloidosis, 1987-2019. N Engl J Med. 2020;382:1567-8.

2. Palladini G, Dispenzieri A, Gertz MA, Kumar S, Wechalekar A, Hawkins PN, et al. New criteria for response to treatment in immunoglobulin light chain amyloidosis based on free light chain measurement and cardiac biomarkers: impact on survival outcomes. J Clin Oncol. 2012;30:4541-9.

3. Manwani R, Cohen O, Sharpley F, Mahmood S, Sachchithanantham S, Foard D, et al. A prospective observational study of 915 patients with systemic AL amyloidosis treated with upfront bortezomib. Blood. 2019;134:2271-80.

4. Godara A, Toskic D, Rosenthal B, Varga C, Kugelmass A, Zhou P, et al. In systemic light-chain amyloidosis complete and very good partial responses are not enough: involved free light chain (iFLC) levels $<10 \mathrm{mg} / \mathrm{L}$ are associated with optimal long-term survival. Blood. 2019;134:4369-4369.

5. Kumar S, Mahmood ST, Lacy MQ, Dispenzieri A, Hayman SR, Buadi FK, et al. Impact of early relapse after auto-SCT for multiple Myeloma. Bone Marrow Transpl. 2008;42:413-20. 2008/09/01

6. Kumar SK, Dispenzieri A, Fraser R, Mingwei F, Akpek G, Cornell R, et al. Early relapse after autologous hematopoietic cell transplantation remains a poor prognostic factor in multiple Myeloma but outcomes have improved over time. Leukemia. 2018;32:986-95. 2018/04/01

7. Corre J, Montes L, Martin E, Perrot A, Caillot D, Leleu X, et al. Early relapse after autologous transplant for Myeloma is associated with poor survival regardless of cytogenetic risk. Haematologica. 2020;105:e480-e483. 06/05

8. Ravichandran S, Cohen Oliver C, Law S, Sachchithanantham S, Mahmood S, Foard $D$, et al. Haematologic responses and survival do not significantly decrease with subsequent lines of therapy in systemic immunoglobulin light chain amyloidosis: results from an analysis of real-world longitudinal data. $\mathrm{Br} J$ Haematol. 2021;194:587-97.

9. Comenzo RL, Reece D, Palladini G, Seldin D, Sanchorawala V, Landau H, et al. Consensus guidelines for the conduct and reporting of clinical trials in systemic light-chain amyloidosis. Leukemia. 2012;26:2317-25. 2012/11/01

10. Palladini G, Milani $P$, Foli $A$, Basset $M$, Russo $F$, Perlini $S$, et al. Presentation and outcome with second-line treatment in $\mathrm{AL}$ amyloidosis previously sensitive to nontransplant therapies. Blood. 2018;131:525-32.

11. Kumar SK, Gertz MA, Lacy MQ, Dingli D, Hayman SR, Buadi FK, et al. Recent improvements in survival in primary systemic amyloidosis and the importance of an early mortality risk score. Mayo Clin Proc. 2011;86:12-18.

12. Muchtar E, Gertz MA, Kourelis TV, Sidana S, Go RS, Lacy MQ, et al. Bone marrow plasma cells $20 \%$ or greater discriminate presentation, response, and survival in AL amyloidosis. Leukemia. 2020;34:1135-43. 2020/04/01

13. Majithia N, Rajkumar SV, Lacy MQ, Buadi FK, Dispenzieri A, Gertz MA, et al. Early relapse following initial therapy for multiple Myeloma predicts poor outcomes in the era of novel agents. Leukemia. 2016;30:2208-13. 2016/11/01
14. Bochtler T, Hegenbart U, Kunz C, Benner A, Kimmich C, Seckinger A, et al. Prognostic impact of cytogenetic aberrations in $\mathrm{AL}$ amyloidosis patients after high-dose melphalan: a long-term follow-up study. Blood. 2016;128:594-602.

15. Kastritis E, Palladini G, Minnema MC, Wechalekar AD, Jaccard A, Lee HC, et al. Daratumumab-based treatment for immunoglobulin light-chain amyloidosis. $\mathrm{N}$ Engl J Med. 2021;385:46-58.

\section{AUTHOR CONTRIBUTIONS}

SR designed the study, collected/analysed the data, and wrote the manuscript. SL, SM, BW, DF, MF, AMN, CW, JDG, HJL \& PNH reviewed and approved the manuscript. ADW supervised the study and approved the manuscript.

\section{COMPETING INTERESTS}

ADW has received honoraria from Janssen, GSK, Celgene, and Takeda. The other authors do not have any conflict of interest to disclose.

\section{ADDITIONAL INFORMATION}

Supplementary information The online version contains supplementary material available at https://doi.org/10.1038/s41375-021-01497-7.

Correspondence and requests for materials should be addressed to Ashutosh D. Wechalekar.

Reprints and permission information is available at http://www.nature.com/ reprints

Publisher's note Springer Nature remains neutral with regard to jurisdictional claims in published maps and institutional affiliations.

(i) Open Access This article is licensed under a Creative Commons Attribution 4.0 International License, which permits use, sharing, adaptation, distribution and reproduction in any medium or format, as long as you give appropriate credit to the original author(s) and the source, provide a link to the Creative Commons license, and indicate if changes were made. The images or other third party material in this article are included in the article's Creative Commons license, unless indicated otherwise in a credit line to the material. If material is not included in the article's Creative Commons license and your intended use is not permitted by statutory regulation or exceeds the permitted use, you will need to obtain permission directly from the copyright holder. To view a copy of this license, visit http://creativecommons. org/licenses/by/4.0/.

(c) The Author(s) 2022 